\title{
Fluctuations of radiation from a chaotic laser below threshold
}

\author{
E. G. Mishchenko \\ Lyman Laboratory, Department of Physics, Harvard University, MA 02138 and \\ L.D. Landau Institute for Theoretical Physics, Moscow 117334, Russia
}

\begin{abstract}
Radiation from a chaotic cavity filled with gain medium is considered. A set of coupled equations describing the photon density and the population of gain medium is proposed and solved. The spectral distribution and fluctuations of the radiation are found. The full noise is a result of a competition between positive correlations of photons with equal frequencies (due to stimulated emission and chaotic scattering) which increase fluctuations, and a suppression due to interaction with a gain medium which leads to negative correlations between photons. The latter effect is responsible for a pronounced suppression of the photonic noise as compared to the linear theory predictions.
\end{abstract}

PACS numbers: 42.50.Ar, 05.40.-a, 42.68.Ay

Introduction. Amplifying random media have now been a focus of intense research for almost a decade $[1,[2,3,4,5]$. The ultimate goal of these efforts is a construction of a random laser in which laser action utilizes the feedback provided by multiple scattering from disorder. Lasing action was reported in a recent work by Cao et al. [6, 7] and Frolov et al. [8]. The difference between the true coherent random laser (RL) and the amplified spontaneous emission (ASE) cannot be identified by the emitted light intensity or its frequency profile as both ASE and RL are characterized by a pronounced narrowing of the spectral linewidth $\gamma$ compared to the atomic transition width $\Gamma$. The knowledge of the statistics of light fluctuations is therefore necessary to make a distinction. While RL action should be coherent and therefore obey the Poissonian distribution, the ASE fluctuations are above the Poissonian level (super-Poissonian noise). Both cases seem to be realized in experiment, that is of RL 7, 9] and ASE [10]. There is still no complete theory able to predict which case ought to be expected in a particular system. From the outset, one expects RL regime to occur whenever there is a single mode above the laser threshold or a few non-overlapping modes, and ASE case to be realized as long as there is a number of overlapping modes above the threshold. In the latter case the scattering between the lasing modes should lead to the enhancement of fluctuations. Since the broadening of the laser modes is given roughly by the inverse mean dwell time $\tau_{d w}^{-1}$, cavity modes overlap strongly provided that the mean level spacing $\Delta$ is small, $\tau_{d w}^{-1} \gg \Delta$. (Hereinafter, we use the units with $\hbar=1$.)

Although there is by now a substantial literature on various models for random lasers [11, 12, 13, 14, 15], we address only those papers that consider fluctuations of laser radiation. Beenakker put forward a quantum approach for a photon statistics based on the random matrix theory [16] for a linear amplifier below laser threshold. The threshold is achieved when the dwell time inside the system $\tau_{d w}$ exceeds the characteristic amplification time determining the rate for photon emission $\tau_{a}$. The threshold features a singularity in the photon density. The fluctuations of radiation demonstrate even stronger singularity as the ratio of the noise power to the mean photon flux $S / \bar{J}$ diverge at the threshold, as a result of scattering between many lasing modes. Linear theory fails upon approaching the threshold when the assumption of equilibrium gain breaks down. Hackenbroich et al. 17] considered statistics of a single lasing mode in a chaotic cavity above the threshold. Cavity opening was assumed to be covered with a barely transparent mirror making the inverse dwell time small compared to the spacing between the modes, $\tau_{d w}^{-1} \ll \Delta$, thus preventing them from overlapping. In Ref. [18] the nonlinear effects have been numerically studied for a system with an opening much smaller than the wavelength. While such a model might be useful for a microwave emission, its relevance to random lasers with typical openings much wider than the wavelength of light remains unclear.

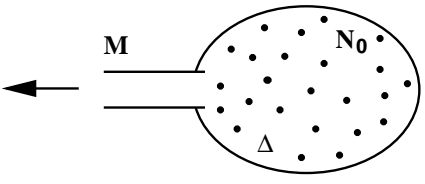

FIG. 1: Chaotic laser: a cavity characterized by the mean level spacing $\Delta$ contains $N_{0}$ particles of a gain medium and is connected to the outside via a waveguide (lead) supporting $M$ transverse channels.

Chaotic Laser. In the present paper we develop a semiclassical approach based on the radiative transfer theory 19] for a chaotic cavity filled with a gain medium, see Fig. 1 (let us, following Refs. 17] and [15], call such a system 'chaotic laser'). The cavity is coupled to the outside through a waveguide supporting $M \gg 1$ transverse modes, so the inverse dwell time $\tau_{d w}^{-1}=\frac{M \Delta}{2 \pi}$ is large compared to the mean level spacing $\Delta=\frac{\pi^{2} c^{3}}{\omega^{2} L^{3}}$, which is inversely proportional to the photon density of states and a system volume $L^{3}$. The ergodic time $\tau_{e r}=L / c$ is the shortest time scale of the system, $\tau_{e r} \ll \tau_{d w}, \tau_{a}$. This condition assures that photons scatter chaotically from the boundaries many times before leaving the cavity or stimulating an emission of another photon, therefore resulting in the homogeneous and isotropic distribution $f_{\omega}$ 
everywhere inside the cavity. The number of photons inside the cavity with the frequency in the interval $d \omega$ is given by $f_{\omega} d \omega / \Delta$. The rate at which this distribution changes in time,

$$
\frac{\partial f_{\omega}}{\partial t}+J_{\omega} \Delta=I_{\omega}
$$

is determined by the photon flux via the waveguide, $J_{\omega}$ and by the photon creation and absorption inside the cavity $I_{\omega}$. The flow of photons through the waveguide (lead) is proportional to the size of the opening (characterized by the number of channels $M$ ) and the difference between the concentration of photons inside and outside the cavity, the latter denoted by $n_{\omega}$ (in general case of external radiation incident on the system),

$$
J_{\omega}=\frac{M}{2 \pi}\left[f_{\omega}-n_{\omega}\right]+\mathcal{J}_{\omega},
$$

with the last term standing for the Langevin source describing fluctuations in the system. The left-hand side of the rate equation (11) has a familiar form of the particle conservation condition while the right-hand side accounts for the photon creation and absorption inside the cavity,

$$
I_{\omega}=w_{\omega}^{+}\left(f_{\omega}+1\right)-w_{\omega}^{-} f_{\omega}+\mathcal{L}_{\omega} \Delta .
$$

The quantities $w_{\omega}^{+}$and $w_{\omega}^{-}$stand for the rates of photon emission and absorption respectively. The probability of photon creation $w_{\omega}^{+}\left(f_{\omega}+1\right)$ takes into account both stimulated and spontaneous emission as required by the quantum statistics of photons.

In addition, Eqs. (13) contain stochastic Langevin sources which are necessary to take into account the randomness of photon transmission through the lead $\mathcal{J}_{\omega}$, and the randomness of the emission and absorption events, $\mathcal{L}_{\omega}$. These terms have zero average, $\overline{\mathcal{J}}_{\omega}=\overline{\mathcal{L}_{\omega}}=0$, and correlators which follow from the assumption that elementary stochastic events of scattering, emission and absorption have independent (and therefore Poissonian) distributions. In particular, the Langevin term associated with non-conservation of particles, $\mathcal{L}_{\omega}=\mathcal{L}_{\omega}^{+}+\mathcal{L}_{\omega}^{-}$ consists of separate contributions from emission and absorption. Different processes have independent distributions (in particular, they are uncorrelated when occur at different times) with the second moments given simply by their average rates (property characteristic for Poissonian processes),

$$
\begin{aligned}
& \overline{\mathcal{L}_{\omega}^{+}(t) \mathcal{L}_{\omega^{\prime}}^{+}\left(t^{\prime}\right)}=\delta_{\omega t} \Delta^{-1} w_{\omega}^{+}\left(\bar{f}_{\omega}+1\right), \\
& \overline{\mathcal{L}_{\omega}^{-}(t) \mathcal{L}_{\omega^{\prime}}^{-}\left(t^{\prime}\right)}=\delta_{\omega t} \Delta^{-1} w_{\omega}^{-} \bar{f}_{\omega}, \quad \overline{\mathcal{L}_{\omega}^{+}(t) \mathcal{L}_{\omega^{\prime}}^{-}\left(t^{\prime}\right)}=0,
\end{aligned}
$$

where $\delta_{\omega t}$ is the shorthand for $\delta\left(\omega-\omega^{\prime}\right) \delta\left(t-t^{\prime}\right)$. Using these formulas we arrive to the following expression,

$$
\overline{\mathcal{L}_{\omega}(t) \mathcal{L}_{\omega^{\prime}}\left(t^{\prime}\right)}=\delta_{\omega t} \Delta^{-1}\left[w_{\omega}^{+}\left(\bar{f}_{\omega}+1\right)+w_{\omega}^{-} \bar{f}_{\omega}\right] .
$$

Similar reasoning allows us to write the correlation function for the Langevin sources describing randomness in the photon flux through the waveguide,

$$
\overline{\mathcal{J}_{\omega}(t) \mathcal{J}_{\omega^{\prime}}\left(t^{\prime}\right)}=\delta_{\omega t} \frac{M}{2 \pi}\left[\bar{n}_{\omega}\left(\bar{n}_{\omega}+1\right)+\bar{f}_{\omega}\left(\bar{f}_{\omega}+1\right)\right] .
$$

Since we assume no reflection in the lead, the only remaining source of fluctuations is the randomness of the photon densities at both ends of the waveguide [20].

In equilibrium at temperature $T$, the function $f_{\omega}$ must be equal to the Bose-Einstein distribution. This fixes the ratio, $w_{\omega}^{-} / w_{\omega}^{+}=e^{\omega / T}$. So far our discussion has been completely general, valid for both the absorbing, $w_{\omega}^{-}>w_{\omega}^{+}(T>0)$, and amplifying $w_{\omega}^{-}<w_{\omega}^{+}(T<0)$, media. Hereinafter we concentrate for simplicity on the case of complete population inversion, $w_{\omega}^{-}=0(T=$ $\left.0^{-}\right)$. The complete population inversion is realized, e.g. when the radiative transition occurs not to a ground state but rather to some excited state with very short (nonradiative) lifetime, see Fig. 2.

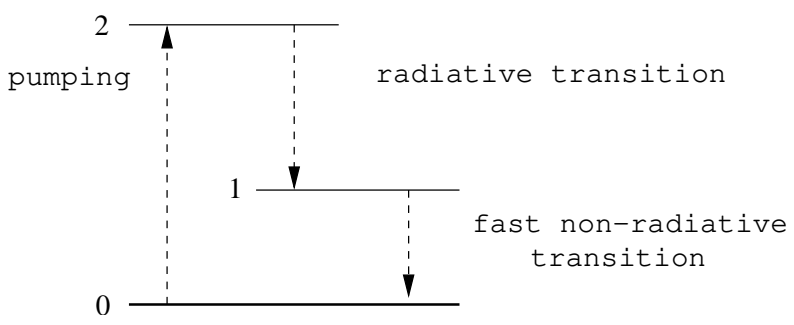

FIG. 2: Complete population inversion realized in a threelevel laser. The atom of the gain medium is pumped to an excited state 2 , the photon is emitted when the atom undergoes a transition to a lower excited state 1 . If the relaxation from the excited state 1 to the ground state 0 is fast, the excited state 1 is almost always empty. Therefore the radiative transition $2 \rightarrow 1$ occurs with the effective temperature $T=0^{-}$.

We assume the spectral line for atomic transitions to be a Lorentzian centered around the frequency $\omega_{0}$ with the linewidth $\Gamma$,

$$
w_{\omega}^{+}=a_{\omega} N \Delta, \quad a_{\omega}=\frac{w_{0} \Gamma}{\pi\left[\left(\omega-\omega_{0}\right)^{2}+\Gamma^{2}\right]}
$$

where $N$ is the number of excited atoms of the gain medium in the cavity, and $w_{0}$ is the total probability (per unit time) for an excited atom to spontaneously emit a photon into vacuum.

The dynamics of the gain medium follows the equation,

$$
\frac{\partial N}{\partial t}=P\left(N_{0}-N\right)-\frac{N}{\tau}-\Delta^{-1} \int d \omega I_{\omega}+\mathcal{N}
$$

where $P$ is the pumping rate, $N_{0}$ is the total number of gain medium particles, and $\tau^{-1}$ is the probability for a non-radiative relaxation. The third term is the total loss from radiative transitions into all possible cavity modes. Note that it contains stochastic emission source, as specified by Eq. (3). The Langevin term $\mathcal{N}$ describes the 
spontaneous fluctuations in the number of excited atoms, which are also assumed Poissonian,

$$
\overline{\mathcal{N}(t) \mathcal{N}\left(t^{\prime}\right)}=\delta\left(t-t^{\prime}\right)\left[P\left(N_{0}-\bar{N}\right)+\bar{N} / \tau\right] .
$$

Note that different Langevin terms $\mathcal{J}_{\omega}, \mathcal{L}_{\omega}, \mathcal{N}$ are independent and have zero cross-correlators. The coupled equations (11), (7) together with the correlators (45), (8) determine the radiation from a chaotic cavity.

Mean photon distribution. We illustrate the above formalism by calculating the average photon flux from the cavity and its fluctuations assuming no radiation incident on the system, $n_{\omega}=0$. In the stationary regime the time-averaged equation for the gain medium (7) is easily solved and the number of excited atoms is expressed via the photon distribution,

$$
\bar{N}=\frac{P N_{0}}{P_{1}+w_{0}+\int d \omega a_{\omega} \bar{f}_{\omega}},
$$

where $P_{1}=P+\tau^{-1}$. Substituting it into Eqs. (1.3) one obtains an integral equation for the photon distribution function, which could be solved analitically. The timeaveraged distribution has the form of a Lorentzian,

$$
\bar{f}_{\omega}=\frac{\Gamma^{2}-\gamma^{2}}{\left(\omega-\omega_{0}\right)^{2}+\gamma^{2}},
$$

with the linewidth $\gamma$ determined from the algebraic equation,

$$
\Gamma^{2}-\gamma^{2}=\frac{2 N_{0} w_{0} \Gamma \gamma P}{M\left(w_{0} \Gamma+P_{1} \gamma\right)}
$$

The total photon flux leaving the cavity $\bar{J}=\frac{M}{2 \pi} \int d \omega \bar{f}_{\omega}$ is found from Eqs. (10,11),

$$
\bar{J}=\frac{N_{0} w_{0} \Gamma P}{w_{0} \Gamma+P_{1} \gamma} .
$$

The cubic equation (11) allows easy numerical solution for any values of the pumping strength $P$ and the number of channels $M$. Here we analyze in detail the more illuminating case of absent non-radiative losses, $\tau^{-1}=0$.

It is convenient to introduce the dimensionless pumping strength and the effective number of radiative transitions,

$$
p=\frac{P}{w_{0}}, \quad \eta=\frac{2 N_{0} w_{0}}{M \Gamma} .
$$

The parameter $\eta$ is the ratio of the photon dwell time in the cavity, $\tau_{d w}=2 \pi / M \Delta$, and the characteristic time of spontaneous photon emission, which at the center of the line is given, according to Eq. (6), by $\tau_{a}=\pi \Gamma / N_{0} w_{0} \Delta$ (if all excited states are filled). The dimensionless width $g=\gamma / \Gamma$ satisfies a simple equation,

$$
1-g^{2}=\frac{\eta p g}{1+p g}
$$

This equation determines the radiation linewidth $g_{\eta}(p)$ as a function of the pumping strength for different system geometries: with the lower values of $\eta$ characterizing open cavities (large number of outgoing channels $M$ ) and the higher values of $\eta$ corresponding to pinching off the waveguide opening. The other two relevant quantities, the population of the gain medium and the outgoing flux can also be expressed in terms of the new dimensionless variables,

$$
\frac{\bar{N}}{N_{0}}=\frac{p g}{1+p g}, \quad \frac{2 \bar{J}}{M \Gamma}=\frac{\eta p}{1+p g} .
$$

For weak pumping, $p \ll 1$, the width is narrowed linearly with the pumping strength,

$$
g_{\eta}(p)=1-\frac{\eta p}{2},
$$

with the narrowing pronounced stronger for less open cavities (larger values of $\eta$ ). The strong pumping, $p \gg 1$, behavior is qualitatively different for different values of the effective number of transitions $\eta$.

(i) Open system, $\eta<1$ (large number of channels supported by the waveguide). The linewidth remains finite for any value of the pumping strength, with,

$$
\lim _{p \rightarrow \infty} g_{\eta}(p)=\sqrt{1-\eta} .
$$

The population of excited atoms becomes saturated, $\bar{N} \rightarrow N_{0}$, while the outgoing flux reaches a constant value $\bar{J} \propto(1-\eta)^{-1 / 2}$. The flux diverges upon pinching off the waveguide opening and approaching the point $\eta=1$. This point is referred to as the 'laser threshold' in the linear amplifier theory [16]. However, it is not a real laser threshold (in a sense discussed towards the end of the paper) but rather a point where nonlinear effects can no longer be disregarded. We call it a 'critical point' here.

(ii) In the critical point, $\eta=1$, the gain population remains full at large pumping rate and the outgoing flux diverges: $\bar{J} \propto p^{1 / 3}$. The linewidth is a slowly decreasing function of pumping $g \propto p^{-1 / 3}$.

(iii) Closed system, $\eta>1$. In this case the linewidth decreases inversely proportional to the pumping intensity. The number of photons leaving the cavity grows linearly with the pumping. The gain population remains partially empty: $\bar{N} / N_{0} \rightarrow 1 / \eta<1$. The results for the strong pumping regime are summarized in Table 1 . The quantitative behavior of the linewidth is illustrated in Fig. 3.

Fluctuations. We now consider the fluctuations of radiation around the mean solution obtained above. Both the photon distribution and the number of excited atoms deviate from their mean values, $f_{\omega}=\bar{f}_{\omega}+\delta f_{\omega}, N=$ $\bar{N}+\delta N$. The fluctuation of the number of excited atoms is found from the linearized equation (7),

$$
\delta N=\bar{N} \frac{\mathcal{N}-\int d \omega \mathcal{L}_{\omega}-\bar{N} \int d \omega a_{\omega} \delta f_{\omega}}{P N_{0}} .
$$


TABLE I: The dependence of the dimensionless linewidth $g=$ $\gamma / \Gamma$, population of the gain medium, and the outgoing photon flux for large values of the pumping intensity $p=P / w_{0} \gg 1$, for different values of the effective number of optic transitions $\eta$ (in the absense of non-radiative losses).

\begin{tabular}{|c|c|c|c|}
\hline$\eta=\frac{2 N_{0} w_{0}}{M \Gamma}$ & $g_{\eta}(p)$ & $\frac{\bar{N}}{N_{0}}$ & $\frac{2 \bar{J}}{M \Gamma}$ \\
\hline$\eta<1$ & $\sqrt{1-\eta}$ & 1 & $\frac{\eta}{\sqrt{1-\eta}}$ \\
\hline$\eta=1$ & $\frac{1}{p^{1 / 3}}$ & 1 & $p^{1 / 3}$ \\
\hline$\eta>1$ & $\frac{1}{(\eta-1) p}$ & $\frac{1}{\eta}$ & $(\eta-1) p$ \\
\hline
\end{tabular}

Substituting it into the equation (11) we obtain the linear

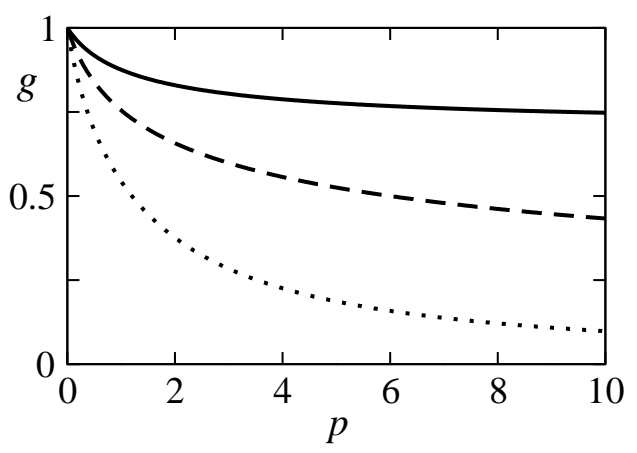

FIG. 3: The linewidth of radiation from a chaotic laser, $g=\gamma / \Gamma$, versus the dimensionless pumping intensity $p=$ $P / w_{0}$ (neglecting non-radiative losses, $\tau^{-1}=0$ ) for different values of the effective number of radiative transitions, $\eta=2 N_{0} w_{0} / M \Gamma$. Solid line: $\eta=1 / 2$, the linewidth $g$ tends to the limit $1 / \sqrt{2} \approx 0.71$ for $p \rightarrow \infty$. Dashed line: $\eta=1$, the linewidth decreases as $g \approx p^{-1 / 3}$. Dotted line: $\eta=2$, the linewidth behaves as $g \approx p^{-1}$.

integral equation for the fluctuation of the distribution function of photons,

$$
\delta f_{\omega}+\frac{M \bar{f}_{\omega}^{2}}{2 \pi a_{\omega} P N_{0}} \int d \omega a_{\omega} \delta f_{\omega}=\frac{\bar{f}_{\omega}\left(\mathcal{L}_{\omega}-\mathcal{J}_{\omega}\right)}{a_{\omega} \bar{N}}+\frac{\bar{f}_{\omega}\left(\bar{f}_{\omega}+1\right)}{P N_{0}}\left(\mathcal{N}-\int d \omega \mathcal{L}_{\omega}\right)
$$

Solving this integral equation is rather straightforward and yields the fluctuation of the outgoing flux,

$$
\delta J_{\omega}=\frac{M}{2 \pi} \delta f_{\omega}+\mathcal{J}_{\omega}=\left(\bar{f}_{\omega}+1\right) \mathcal{L}_{\omega}-\bar{f}_{\omega} \mathcal{J}_{\omega}-A_{\omega} \int d \omega^{\prime}\left[\left(\bar{f}_{\omega^{\prime}}+1\right) \mathcal{L}_{\omega^{\prime}}-\bar{f}_{\omega^{\prime}} \mathcal{J}_{\omega^{\prime}}\right]+A_{\omega} \mathcal{N}
$$

In the last expression we utilized the following notations,

$$
A_{\omega}=\frac{M \bar{f}_{\omega}\left(\bar{f}_{\omega}+1\right)}{2 \pi P N_{0} D}, \quad D=1+\frac{M}{2 \pi P N_{0}} \int d \omega \bar{f}_{\omega}^{2}
$$

By using the correlation functions of the Langevin sources (4)-(5) and (8) the frequency-resolved noise power, $S_{\omega \omega^{\prime}}=\int d t \overline{J_{\omega}(t) J_{\omega^{\prime}}(0)}$, is found,

$$
\begin{aligned}
S_{\omega \omega^{\prime}}= & \delta\left(\omega-\omega^{\prime}\right) \widetilde{S}_{\omega}-\left(A_{\omega} \widetilde{S}_{\omega^{\prime}}+A_{\omega^{\prime}} \widetilde{S}_{\omega}\right) \\
& +A_{\omega} A_{\omega^{\prime}}\left[\widetilde{S}+P\left(N_{0}-\bar{N}\right)+\tau^{-1} \bar{N}\right],
\end{aligned}
$$

where we introduced the notations,

$$
\widetilde{S}_{\omega}=\bar{J}_{\omega}\left(\bar{f}_{\omega}+1\right)\left(\bar{f}_{\omega}^{2}+\bar{f}_{\omega}+1\right), \quad \widetilde{S}=\int d \omega \widetilde{S}_{\omega}
$$

The first term in Eq. (20) represents the correlations between the photons of the same frequency. Those correlations are the result of spontaneous and stimulated emission and also due to chaotic photon scattering inside the cavity. Assuming again the absence of non-radiative losses we can express the Fano factor (the ratio of the noise power to the mean flux) at the center of the line, $\omega=\omega_{0}$, via a single parameter,

$$
F_{0}=\frac{S_{\omega_{0}}}{\bar{J}_{\omega_{0}}}=\frac{1-g^{2}+g^{4}}{g^{6}}
$$

namely the dimensionless linewidth $g=\gamma / \Gamma$. This is a monotonously increasing function of the pumping strength $p$. As before, we analyze three possible regimes, making use of the above results summarized in Table 1.

(i) Open system, $\eta<1$. When the pumping intensity increases, $p \rightarrow \infty$, the Fano factor approaches the finite value,

$$
F_{0}=\frac{1-\eta+\eta^{2}}{(1-\eta)^{3}}
$$

This expression reproduces the result of the random matrix theory [16] for a linear amplifier with a complete 
population inversion. Indeed, in an open system, $\eta<1$, the gain medium dynamics becomes suppressed for large values of the pumping strength when all excited states become occupied.

(ii) Critical point, $\eta=1$. For large pumping intensity, $p \gg 1$, the Fano factor grows quadratically,

$$
F_{0}=p^{2} .
$$

(i) Finally, in a closed system, $\eta>1$, the Fano factor behaves as the sixth power of the pumping strength $p$ for $p \gg 1$,

$$
F_{0}=(\eta-1) p^{6}
$$

The quantitative behavior of the Fano factor (22) is illustrated in Fig. 4 where numerical results are presented.

Cross-correlations. Contrary to the linear theory predicting the photons of different frequencies to be uncorrelated, we observe significant off-diagonal correlations, as attributed by the expression (20). This is due to the strong coupling of photons to the fluctuations of the gain medium. The important fact is that these correlations are negative. It could be understood from the following reasoning: the emission of a photon at some frequency reduces the population of excited atoms making therefore the emission of another photon less likely.

To illustrate better the role of cross-correlations it is useful to calculate the noise power for the total radiation from the cavity. Integrating the expression (20) over both frequencies yields the noise power $S=\int d \omega d \omega^{\prime} S_{\omega \omega^{\prime}}$ for the total photon flux leaving the system,

$$
S=\bar{J} A^{2}\left(1+\frac{2 \gamma}{w_{0} \Gamma \tau}\right)+\widetilde{S}(1-A)^{2},
$$

here $A=\int d \omega A_{\omega}$. By using the expressions (9)-(10) for the mean distributions, the necessary integrals are easily evaluated,

$$
\begin{aligned}
& \widetilde{S}=\frac{M\left(\Gamma^{2}-\gamma^{2}\right)}{32 \gamma^{7}}\left(5 \Gamma^{6}-3 \Gamma^{4} \gamma^{2}+7 \Gamma^{2} \gamma^{4}+7 \gamma^{6}\right), \\
& A=\frac{M\left(\Gamma^{4}-\gamma^{4}\right)}{4 P N_{0} \gamma^{3} D}, \quad D=1+\frac{M\left(\Gamma^{2}-\gamma^{2}\right)^{2}}{4 P N_{0} \gamma^{3}}
\end{aligned}
$$

Again, we analyze the obtained general expression neglecting non-radiative losses, $\tau^{-1}=0$. By using the dimensionless variables $p$ and $\eta$ defined in the preceding sections, the Fano factor for the total photon flux leaving the cavity, $F=S / \bar{J}$, can be written as,

$$
F=\frac{4\left(1+g^{2}\right)^{2}+p^{2}\left(5-3 g^{2}+7 g^{4}+7 g^{6}\right)}{4\left(1+g^{2}+2 p g^{3}\right)^{2}}
$$

The dimensionless width $g$ is a function of both the pumping strength $p$ and the number of optic transitions $\eta$ and is given by the solution of the equation (13). The dependence of the Fano factor (25) on the pumping strength is plotted in Fig. 5. A surprising feature of this dependence is the suppression of the noise below Poissonian level for intermediate values of the pumping strength. This is the demonstration of negative correlations between the photons due to their interaction with the gain media. Indeed, despite the fact that the diagonal correlations 21,22 increase monotonously with the pumping $p$ as a result of photon bunching, the total flow of outgoing photons becomes less fluctuating. For large values of $p$ the enhancement of the photon bunching overwhelms the negative correlations and the Fano factor $F$ increases. Still, it remains strongly reduced compared to the frequency-resolved Fano factor (22), see Fig. 5.

(i) For an open system, $\eta<1$, and large values of the pumping strength the Fano factor approaches the limit,

$$
F=\frac{1-2 \eta+\frac{7}{4} \eta^{2}-\frac{7}{16} \eta^{3}}{(1-\eta)^{3}}
$$

and diverges at the critical point $\eta \rightarrow 1$. Its ratio, $F / F_{0}$, to the frequency-resolved Fano factor (23) remains constant and decays monotonously from 1 to $5 / 16$ while $\eta$ changes from 0 to 1 .

(ii) Critical point, $\eta=1$. The Fano factor increases quadratically for large pumping, $p \gg 1$,

$$
F=\frac{5 p^{2}}{36}
$$

with the ratio of the two factors remaining finite, $F / F_{0} \rightarrow$ $5 / 36$.

(iii) Finally, in a closed system, $\eta>1$, the fluctuations of the total flux are strongly suppressed,

$$
F=\frac{5 p^{2}}{4}
$$

compared to the diagonal fluctuations, with the ratio $F / F_{0} \rightarrow 0$.

Laser threshold. So far the mean level spacing $\Delta$ has not entered the expressions for the mean flux nor the noise power. However, it sets the condition for applicability of the entire approach. The above equations are semiclassical and imply incoherent radiation from the system. This is so provided that there are many overlapping modes inside the cavity within the linewidth $\gamma$. A characteristic mode width is given by the inverse dwell time $\tau_{d w}^{-1}=\frac{M \Delta}{2 \pi}$. As long as $\gamma, \tau_{d w}^{-1} \gg \Delta$, the mode overlapping is strong. Estimating the linewidth as $\gamma \approx \Gamma / \eta p$ (for $\eta>1$ ), we can establish the necessary conditions: $\frac{\Gamma^{2} M}{P N_{0}} \gg \Delta$, and $M \gg 1$.

Another condition for the semiclassical theory to be valid requires the system to remain below the laser threshold. The latter condition, for the $i$-th cavity mode, is determined by the relation, $\tau_{d w}^{(i)} / \tau_{a}^{(i)} \equiv s^{(i)}=1$, with the amplification time $\tau_{a}^{(i)}$ being in general a function of the photon population of all cavity modes and, hence, some complicated function of the pumping strength. From our equations (6) and (9) the semiclassical value of $s$ at the center of the line $\omega=\omega_{0}$ is found,

$$
\bar{s}=\frac{\tau_{d w}}{a_{\omega_{0}} \bar{N} \Delta}=1-g^{2}<1,
$$


and the system stays below the threshold for any pumping intensity. However, as the pumping increases, $g \rightarrow 0$, the quantum distribution of $s^{(i)}$ around the mean value $\bar{s}$ becomes important 21]. At some critical pumping, the mode with the largest $s^{(i)}$ reaches its threshold value and starts to lase in a coherent fashion. This regime is beyond the reach of the semiclassical theory. The problem of fluctuations of radiation from a chaotic laser above the threshold in the non-linear quantum regime remains an open one.

Conclusions. We presented a semiclassical theory of the incoherent radiation from a chaotic cavity containing optically active medium based on the radiative transfer theory with Langevin sources. The theory reproduces the results of the random matrix approach for fluctuations of radiation from a linear amplifier. By accounting for the dynamics of the gain media the theory is extended to incorporate the nonlinear effects: in particular, the spectral line narrowing of the emerging light is calculated. The fluctuations of radiation from the system are the result of two competing effects: the enhancement of fluctuations due to photon bunching and the suppression of fluctuations due to interaction with the gain medium.

I gratefully acknowledge fruitful discussions with C. Beenakker and B. Halperin. Conversations with A. Brataas and Ya. Tserkovnyak are also appreciated. This work was supported by the National Science Foundation Grant DMR-02-33773 and by the Russian Foundation for Basic Research Grant 01-02-16211.
[1] N.M. Lawandy, R.M. Balachandran, A.S.L. Gomes, and E. Sauvain, Nature (London) 368, 436 (1994).

[2] W.L. Sha, C.-H. Liu, and R.R. Alfano, Opt. Lett. 19, 1922 (1994).

[3] D.S. Wiersma, M.P. van Albada, and A. Lagendijk, Nature (London) 373, 203 (1995).

[4] D.Z. Zhang, B.Y. Cheng, J.H. Yang, Y.L. Zhang, W. Hu, and Z.L. Li, Opt. Commun. 118, 462 (1995).

[5] C. Gmachl, F. Capasso, E.E. Narimanov, J.U. Nökel, A.D. Stone, J. Faist, D.L. Sivco, and A.Y. Cho, Science 280, 1556 (1998).

[6] H. Cao, Y.G. Zhao, S.T. Ho, E.W. Seelig, Q.H. Wang, and R.P.H. Chang, Phys. Rev. Lett. 82, 2278 (1999).

[7] H. Cao, Y. Ling, J.Y. Xu, C.Q. Cao, and P. Kumar, Phys. Rev. Lett. 86, 4524 (2001).

[8] S.V. Frolov, Z.V. Vardeny, K. Yoshino, A. Zakhidov, and R.H. Baughman, Phys. Rev. B 59, 5284(R) (1999); S.V. Frolov, Z.V. Vardeny, A.A. Zakhidov, and R.H. Baughman, Opt. Commun. 162, 241 (1999).

[9] R.C. Polson, A. Chipouline, and Z.V. Vardeny, Adv. Mater. 13, 760 (2001).

[10] G. Zacharakis, N.A. Papadogiannis, G. Fillippidis, and T.G. Papazoglou, Opt. Lett. 25, 923 (2000).

[11] X. Jiang and C.M. Soukoulis, Phys. Rev. Lett. 85, 70 (2000); Phys. Rev. E 65, 025601 (2002).

[12] C. Vanneste and P. Sebbah, Phys. Rev. Lett. 87, 183903 (2001).

[13] A.L. Burin, M.A. Ratner, H. Cao, and R.P.H. Chang, Phys. Rev. Lett. 87, 215503 (2001); 88, 093904 (2002).

[14] V.M. Apalkov, M.E. Raikh, and B. Shapiro, Phys. Rev. Lett. 89, 016802 (2002).

[15] L.I. Deych, Phys. Rev. B 67, 161101(R) (2003).

[16] C.W.J. Beenakker, Phys. Rev. Lett. 81, 1829 (1998); also in Diffusive Waves in Complex Media, edited by J.-P. Fouque, NATO ASI C531 (Kluwer, Dordrecht, 1999).

[17] G. Hackenbroich, C. Viviescas, B. Elattari, and F. Haake, Phys. Rev. Lett. 86, 5262 (2001).

[18] M. Patra, Phys. Rev. A 65, 043809 (2002).

[19] E.G. Mishchenko and C.W.J. Beenakker, Phys. Rev. Lett. 83, 5475 (1999).

[20] In a more general case when photons get reflected in the waveguide with the probability $R$ (due to some sort of opacity or scattering from defects of the boundaries), the correlator of Langevin sources takes the form, $\overline{\mathcal{J}_{\omega}(t) \mathcal{J}_{\omega^{\prime}}\left(t^{\prime}\right)}=$ $\delta_{\omega t} \frac{M}{2 \pi}\left\{(1-R)^{2}\left[\bar{n}_{\omega}\left(\bar{n}_{\omega}+1\right)+\bar{f}_{\omega}\left(\bar{f}_{\omega}+1\right)\right]+R(1-R)\right.$ $\left.\times\left[\bar{n}_{\omega}\left(\bar{f}_{\omega}+1\right)+\bar{f}_{\omega}\left(\bar{n}_{\omega}+1\right)\right]\right\}$.

[21] T.Sh. Misirpashaev and C.W.J. Beenakker, Phys. Rev. B 57, 2041 (1998). 


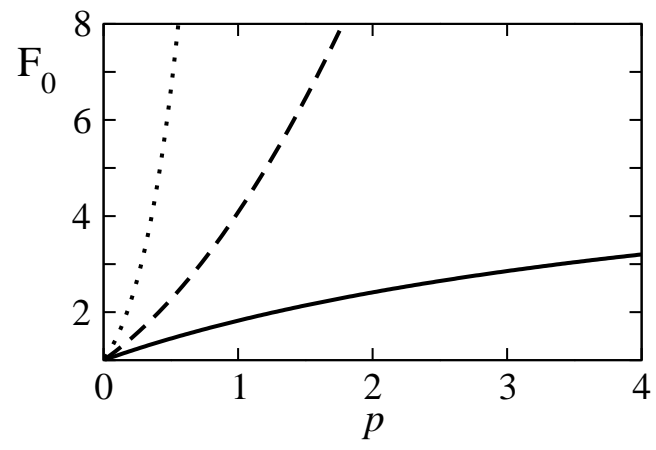

FIG. 4: The Fano factor for the fluctuations of radiation at the center of the line, $\omega=\omega_{0}$, versus the dimensionless pumping intensity (neglecting non-radiative losses) for different values of the effective number of radiative transitions: $\eta=1 / 2$ (solid line), 1 (dashed line), 2 (dotted line).

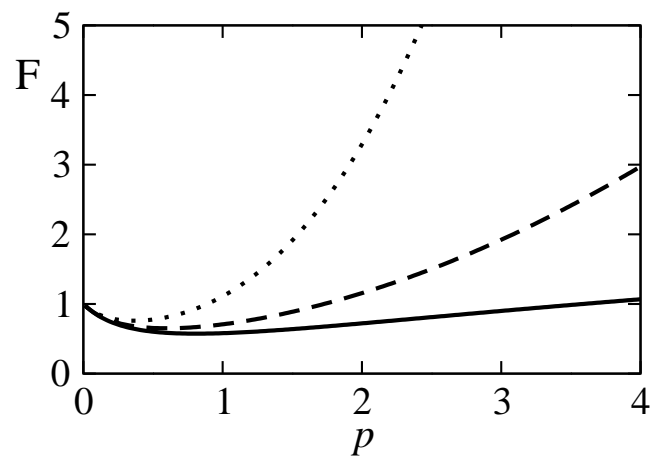

FIG. 5: The Fano facor for the total radiation from a chaotic laser for different values of the effective number of radiative transitions: $\eta=1 / 2$ (solid line), 1 (dashed line), 2 (dotted line). 